\title{
NONEXISTENCE OF POSITIVE FINITE MORSE INDEX SOLUTIONS TO AN ELLIPTIC PROBLEM WITH SINGULAR NONLINEARITY*
}

\author{
YUXIA GUO ${ }^{\dagger}$ AND JUNCHENG WEI ${ }^{\ddagger}$
}

Abstract. We consider the following elliptic problem

$$
\Delta u=|y|^{\alpha} u^{\tau} \text { in } \mathbb{R}^{\mathbb{N}},
$$

where $N \geq 2, \alpha \geq 0$ and $\tau<0$. We prove that if $2 \leq N<2+\frac{2(2+\alpha)}{\tau-1}(\tau-\sqrt{\tau(\tau-1)})$, there is no positive smooth solution with finite Morse index.

Key words. Positive solution, Finite Morse index, Singular nonlinearity.

AMS subject classifications. 35B45, 35J25

1. Introduction. We consider the positive smooth solutions of the following equation:

$$
\Delta u=|y|^{\alpha} u^{\tau} \text { in } \mathbb{R}^{\mathbb{N}} \text {, with } N \geq 2, \alpha \geq 0, \tau<0 .
$$

The motivation to study equation (1.1) comes the fact that this equation appears in many branches of applied science, such as in mechanics and in physics. In particular, it can be the steady states of thin films. In the literatures, the following equations

$$
u_{t}=-\nabla \cdot(f(u) \nabla \Delta u)-\nabla \cdot(g(u) \nabla u)
$$

have been used to model the dynamics of thin films of viscous fluids, where $u(x, t)$ is the height of the air (liquid) interface. The zero set $\sum_{u}=\{u=0\}$ is the liquid (solid) interface and is sometimes called set of ruptures, which plays a very important role in the study of thin films. The coefficient $f(u)$ reflects surface tension effects, a typical choice is $f(u)=u^{3}$. And the coefficient $g(u)$ of the second-order term reflects additional forces such as gravity $g(u)=u^{3}$, and Van der Waals interactions $g(u)=u^{m}, m<0$. For more information on the background of the equation (1.2), we refer to $[2,3,19,20]$ and the references therein. If we choose $f(u)=u^{l}, g(u)=u^{m}$, where $l, m \in \mathbb{R}$, and we consider the steady state of (1.2), we see that if $u$ satisfies the following equation

$$
u^{l} \nabla \Delta u+u^{m} \nabla u=\mathcal{C}
$$

then $u$ is a steady state of $(1.2)$, where $\mathcal{C}=\left(C_{1}, C_{2}, \ldots, C_{n}\right)$ is some constant vector. By assuming $\mathcal{C}=0$ (which prevents linear terms on $x$ ), we obtain

$$
\Delta u+\frac{u^{k}}{k}-C=0
$$

with $k=m-l+1$ and $C$ is some constant. Here we have assumed that $k \neq 0$, otherwise we replaced $\frac{u^{k}}{k}$ by $\ln u$. If we choose $f(u)=u^{3}, g(u)=-u^{m}$ with $m<1$,

\footnotetext{
*Received May 20, 2008; accepted for publication October 21, 2008.

${ }^{\dagger}$ Department of Mathematics, Tsinghua University, Beijing, 100084, P. R. China (yguo@math. tsinghua.edu.cn).

${ }_{\ddagger}$ Department of Mathematics, The Chinese University of Hong Kong, Shatin, Hong Kong (wei@math.cuhk.edu.hk).
} 
and take $k=m-2<-1$. Then by choosing $C=0$ and a simple scaling we get the equation

$$
\Delta u=u^{p}, p<-1 .
$$

Note that solutions to (1.1) can explain the behaviors of steady states for equation (1.2) in some special cases but the reverse is not true. We mention that when $\tau=-2$, the equation (1.1) also arises in the study of a simple electrostatic MicroElectromechanical System (MEMS) device consisting of a thin dielectric elastic membrane with boundary supported at 0 below a rigid plate located at +1 . We refer to $[6,9,10,11,14,15]$ and the references therein. When $\tau=-1$, our equation is related to the study of singular minimal hypersurfaces with symmetry, see [21, 24] and references therein.

When $\Omega=\mathbb{R}^{\mathbb{N}}$, the radially symmetry solutions to (1.3) has been studied in [14]. The quantitative properties of solutions to (1.1) including the gradient estimates, $L^{1}-$ estimates, global upper bounds, Liouville properties, classifications of stable solutions and symmetry properties have been studied in [22];

From the variational point of view, it is also very interesting to discuss positive solution with finite Morse index to (1.1). The nonexistence results of finite morse index solutions in an unbounded domain plays a crucial role to obtain a priori $L^{\infty}$ bounds for solutions of semilinear boundary value problems in bounded domain (see [12] for the case of positive solutions and [1] for solutions with finite Morse index). On the other hand, nonlinear forms of Liouville type's results, combined with degree type arguments, are very useful to obtain the existence of solutions of semilinear boundary value problem in bounded domain (see for instance [8]). In this paper, we are going to study the nonexistence of finite Morse index solutions in $\mathbb{R}^{\mathbb{N}}$. Assume that $u \in C^{2}\left(\mathbb{R}^{\mathbb{N}}\right)$ is a positive solution to (1.1), we define

$$
E(\varphi)=\int_{\mathbb{R}^{N}}\left(|\nabla \varphi|^{2}+\tau|y|^{\alpha} u^{\tau-1} \varphi^{2}\right), \varphi \in C_{c}^{1}\left(\mathbb{R}^{N}\right) .
$$

By definition, we say that the positive solution $u$ to (1.1) with finite Morse index $K \geq 1$ if there exist $L^{2}$ orthogonal nontrivial functions $\left\{\varphi_{j}\right\}_{j=1}^{K} \subset C_{c}^{1}\left(\mathbb{R}^{\mathbb{N}}\right)$ such that $E(\varphi)<0$ for $\varphi \in W:=\operatorname{span}\left\{\varphi_{j}\right\} \backslash\{\theta\}$, and $E(\varphi) \geq 0$ for $\varphi \perp W$; We say that the solution $u$ is stable if $E(\varphi) \geq 0$ for any $\varphi \in C_{c}^{1}\left(\mathbb{R}^{\mathbb{N}}\right)$; We say that the solution is stable outside of a compact set $\Omega \subset \mathbb{R}^{\mathbb{N}}$ if $E(\varphi) \geq 0$ for any $\varphi \in C_{c}^{1}\left(\mathbb{R}^{\mathbb{N}} \backslash \Omega\right)$.

REMARK 1.1. From the above definitions, one can easily see that any finite Morse index solution $u$ is stable outside of a compact set $\Omega$. In fact, assume that $u$ is a solution with finite Morse index $K \geq 1$ such that $E(\varphi)<0$ for any $\varphi \in$ $W=\operatorname{span}\left\{\varphi_{1}, \ldots, \varphi_{k}\right\} \subset C_{c}^{1}\left(\mathbb{R}^{\mathbb{N}}\right)$. Hence $E(\bar{\psi}) \geq 0$ for any $\psi \in C_{c}^{1}\left(\mathbb{R}^{\mathbb{N}} \backslash \Omega\right)$, where $\Omega:=\bigcup_{j=1}^{K} \operatorname{supp}\left(\varphi_{i}\right)$.

Our main results are:

TheOREM 1.2. If $2 \leq N \leq 2+\frac{2(2+\alpha)}{\tau-1}(\tau-\sqrt{\tau(\tau-1)})$, then there are no finite Morse index positive solutions to (1.1) in $\mathbb{R}^{\mathbb{N}}$.

Remark 1.3. The case of $\alpha=0, N=2$ has been proved by Ma and Wei ([22]).

REMARK 1.4. Esposito ([5]) considered the following problem

$$
\Delta u=\lambda|x|^{\alpha} u^{-2} \text { in } \Omega, u=1 \text { on } \partial \Omega
$$


where $\Omega$ is a bounded domain. Result similar to Theorem 1.2 was proved.

REMark 1.5. Farina ([7]) proved that there are no finite Morse index solution to the purely critical exponent

$$
\Delta u+u^{p}=0, u>0 \text { in } R^{N}
$$

when $1<p<p_{J L}$, where $p_{J L}$ is the Joseph-Lungren exponent. Dancer and Farina [4] extended the result to exponential nonlinearity. We shall follow the proofs in [4] and [7].

The paper is organized as follows. In section 2, for simple and clear reason, we first prove some inequalities for the solution of the equation (1.1) with $\alpha=0$, which are crucial for the present work. In Section 3, we prove Theorem 1.2 with $\alpha=0$ by a contrary argument. Finally, in Section 4 , we discuss the general equation with $\alpha \geq 0$.

Acknowledgements. This paper was done while the first author was visiting the Department of Mathematics, Chinese University of Hong Kong. She would like to thank the Department for its hospitality. She also thanks Professor Dong Ye for many useful discussions. The second author thanks Professor Dancer for many interesting discussions.

2. Preliminaries. In this section, we are going to prove some inequalities for the positive solutions of the following equation:

$$
\Delta u=u^{\tau}, \text { in } \mathbb{R}^{\mathbb{N}}, N \geq 2, \tau<0 .
$$

Our first result is :

Proposition 2.1. Let $\Omega$ be a domain (bounded or not) of $\mathbb{R}^{\mathbb{N}}$. Suppose that $u$ is a positive stable solution of (2.1), then for any $\gamma \in[1,1-2 \tau+2 \sqrt{\tau(\tau-1)})$ and any integer $m \geq \max \left\{\frac{\tau-\gamma}{\tau-1}, 2\right\}$, there exists a constant $C=C(\tau, m, \gamma)>0$ depending on $\tau, m$ and $\gamma$ such that

$$
\int_{\Omega}\left(\left|\nabla\left(u^{\frac{-\gamma+1}{2}}\right)\right|^{2}+u^{\tau-\gamma}\right) \psi^{2 m} \leq C(\tau, m, \gamma) \int_{\Omega}\left(|\nabla \psi|^{2}+|\psi \| \Delta \psi|\right)^{\frac{\tau-\gamma}{\tau-1}},
$$

for any test functions $\psi \in C_{c}^{2}(\Omega)$ satisfying $|\psi| \leq 1$ in $\Omega$.

Proof. The proof is consist in the following steps.

Step 1: We prove for any $\varphi \in C_{c}^{2}(\Omega)$

$$
\int_{\Omega}\left(\left|\nabla\left(u^{\frac{-\gamma+1}{2}}\right)\right|^{2} \varphi^{2}=\frac{(-\gamma+1)^{2}}{4 \gamma} \int_{\Omega} u^{\tau-\gamma} \varphi^{2}-\frac{(-\gamma+1)}{4 \gamma} \int_{\Omega} u^{-\gamma+1} \Delta\left(\varphi^{2}\right) .\right.
$$

To prove (2.2), we multiply the equation $\Delta u=u^{\tau}$ by $u^{-\gamma} \varphi^{2}$ and integrate by parts to obtain

$$
\gamma \int_{\Omega}|\nabla u|^{2} u^{-\gamma-1} \varphi^{2}-\int_{\Omega} \nabla u \nabla\left(\varphi^{2}\right) u^{-\gamma}=\int_{\Omega} u^{\tau-\gamma} \varphi^{2}
$$

This deduces that

$$
\begin{aligned}
& \frac{\gamma}{\left(\frac{-\gamma+1}{2}\right)^{2}} \int_{\Omega}\left|\nabla\left(u^{\frac{-\gamma+1}{2}}\right)\right|^{2} \varphi^{2}-\int_{\Omega} \nabla\left(\frac{u^{-\gamma+1}}{-\gamma+1}\right) \nabla\left(\varphi^{2}\right) \\
= & \frac{\gamma}{\left(\frac{-\gamma+1}{2}\right)^{2}} \int_{\Omega}\left|\nabla\left(u^{\frac{-\gamma+1}{2}}\right)\right|^{2} \varphi^{2}+\int_{\Omega}\left(\frac{u^{-\gamma+1}}{-\gamma+1}\right) \Delta\left(\varphi^{2}\right) \\
= & \int_{\Omega} u^{\tau-\gamma} \varphi^{2} .
\end{aligned}
$$


Hence we obtain, by multiplying the constant $\frac{(-\gamma+1)^{2}}{4 \gamma}$ on the two sides of the above last equality,

$$
\int_{\Omega}\left(\left|\nabla\left(u^{\frac{-\gamma+1}{2}}\right)\right|^{2} \varphi^{2}=\frac{(-\gamma+1)^{2}}{4 \gamma} \int_{\Omega} u^{\tau-\gamma} \varphi^{2}-\frac{(-\gamma+1)}{4 \gamma} \int_{\Omega} u^{-\gamma+1} \Delta\left(\varphi^{2}\right) .\right.
$$

Step 2: We prove that for any $\varphi \in C_{c}^{2}(\Omega)$

$$
\left(-\tau-\frac{(-\gamma+1)^{2}}{4 \gamma}\right) \int_{\Omega} u^{\tau-\gamma} \varphi^{2} \leq \int_{\Omega} u^{-\gamma+1}|\nabla \varphi|^{2}-\left(\frac{(-\gamma+1)}{4 \gamma}+\frac{1}{2}\right) \int_{\Omega} u^{-\gamma+1} \Delta\left(\varphi^{2}\right) .
$$

Since $u$ is stable, by definition, we have for any $\varphi \in C_{c}^{1}(\Omega)$

$$
\int_{\Omega}|\nabla \varphi|^{2}+\tau u^{\tau-1} \varphi^{2} \geq 0
$$

Note that for any $\varphi \in C_{c}^{2}(\Omega), \psi=u^{\frac{-\gamma+1}{2}} \varphi \in C_{c}^{1}(\Omega)$, thus we can use it as a test function in the above inequality, then we obtain :

$$
\begin{aligned}
-\tau \int_{\Omega} u^{\tau-\gamma} \varphi^{2} & \leq \int_{\Omega}\left|\nabla\left(u^{\frac{-\gamma+1}{2}} \varphi\right)\right|^{2} \\
& =\int_{\Omega}\left|\nabla\left(u^{\frac{-\gamma+1}{2}}\right)\right|^{2} \varphi^{2}+\int_{\Omega}\left(u^{\frac{-\gamma+1}{2}}\right)^{2}|\nabla \varphi|^{2}+\int_{\Omega} 2 \nabla\left(u^{\frac{-\gamma+1}{2}}\right) \nabla \varphi u^{\frac{-\gamma+1}{2}} \varphi \\
& =\int_{\Omega}\left|\nabla\left(u^{\frac{-\gamma+1}{2}}\right)\right|^{2} \varphi^{2}+\int_{\Omega}\left(u^{\frac{-\gamma+1}{2}}\right)^{2}|\nabla \varphi|^{2}-\frac{1}{2} \int_{\Omega} u^{-\gamma+1} \Delta\left(\varphi^{2}\right) .
\end{aligned}
$$

Inserting the formula (2.2) into the above last inequality, we get

$$
\begin{aligned}
-\tau \int_{\Omega} u^{\tau-\gamma} \varphi^{2} \leq & \frac{(-\gamma+1)^{2}}{4 \gamma} \int_{\Omega} u^{\tau-\gamma} \varphi^{2}-\frac{-\gamma+1}{4 \gamma} \int_{\Omega} u^{-\gamma+1} \Delta\left(\varphi^{2}\right) \\
& +\int_{\Omega} u^{-\gamma+1}|\nabla \varphi|^{2}-\frac{1}{2} \int_{\Omega} u^{-\gamma+1} \Delta\left(\varphi^{2}\right) .
\end{aligned}
$$

It follows that

$$
\left(-\tau-\frac{(-\gamma+1)^{2}}{4 \gamma}\right) \int_{\Omega} u^{\tau-\gamma} \varphi^{2} \leq \int_{\Omega} u^{-\gamma+1}|\nabla \varphi|^{2}-\left(\frac{(-\gamma+1)}{4 \gamma}+\frac{1}{2}\right) \int_{\Omega} u^{-\gamma+1} \Delta\left(\varphi^{2}\right)
$$

Step 3: We prove that for any $\gamma \in[1,1-2 \tau+2 \sqrt{\tau(\tau-1)})$ and any integer $m \geq\left\{\frac{\tau-\gamma}{\tau-1}, 2\right\}$ there exists a constant $C=C(\tau, m, \gamma)$ such that for any $\psi \in C_{c}^{2}(\Omega)$ satisfying $|\psi| \leq 1$ in $\Omega$, it holds that

$$
\begin{gathered}
\int_{\Omega} u^{\tau-\gamma} \psi^{2 m} \leq C(\tau, m, \gamma) \int_{\Omega}\left(|\nabla \psi|^{2}+|\psi||\Delta \psi|\right)^{\frac{\tau-\gamma}{\tau-1}}, \\
\int_{\Omega}\left|\nabla\left(u^{\frac{-\gamma+1}{2}}\right)\right|^{2} \psi^{2 m} \leq C(\tau, m, \gamma) \int_{\Omega}\left(|\nabla \psi|^{2}+|\psi||\Delta \psi|\right)^{\frac{\tau-\gamma}{\tau-1}} .
\end{gathered}
$$

Moreover, the constants $C(\tau, m, \gamma)$ can be explicitly computed.

Indeed, from (2.3), we have for any $\varphi \in C_{c}^{2}(\Omega)$

$$
\alpha \int_{\Omega} u^{\tau-\gamma} \varphi^{2} \leq \int_{\Omega} u^{-\gamma+1}|\nabla \varphi|^{2}+\beta \int_{\Omega} u^{-\gamma+1} \varphi \Delta \varphi
$$


where $\alpha=\left(-\tau-\frac{(-\gamma+1)^{2}}{4 \gamma}\right) \geq 0, \beta=-\left(\frac{(-\gamma+1)}{4 \gamma}+\frac{1}{2}\right)<0$. Now for any $\psi \in C_{c}^{2}(\Omega)$ with $|\psi| \leq 1$ in $\Omega$. Let $\varphi=\psi^{m}$, where $m \geq \max \left\{\frac{\tau-\gamma}{\tau-1}, 2\right\}$, then $\varphi \in C_{c}^{2}(\Omega)$. Hence we have:

$$
\begin{aligned}
\alpha \int_{\Omega} u^{\tau-\gamma} \psi^{2 m} & \leq \int_{\Omega} u^{-\gamma+1}\left|\nabla\left(\psi^{m}\right)\right|^{2}+\beta \int_{\Omega}|u|^{-\gamma+1} \psi^{m} \Delta \psi^{m} \\
& =\int_{\Omega} u^{-\gamma+1} \psi^{2 m-2}\left[m^{2}|\nabla \psi|^{2}+\beta m(m-1)|\nabla \psi|^{2}+\beta m \psi \Delta \psi\right] .
\end{aligned}
$$

It follows that

$$
\int_{\Omega} u^{\tau-\gamma} \psi^{2 m} \leq C_{1} \int_{\Omega} u^{-\gamma+1}|\psi|^{2 m-2}\left[|\nabla \psi|^{2}+|\psi \Delta \psi|\right],
$$

with $C_{1}=\frac{m^{2}+\beta m(m-1)}{\alpha}>-\frac{\beta m}{\alpha} \geq 0$.

Note that $m \geq \max \left\{\frac{\tau-\gamma}{\tau-1}, 2\right\}$, and $(2 m-2)\left(\frac{\tau-\gamma}{1-\gamma}\right) \geq 2 m$, hence $|\psi|^{(2 m-2)\left(\frac{\tau-\gamma}{1-\gamma}\right)} \leq$ $|\psi|^{2 m}$. By using Hölder inequality, we have:

$$
\begin{aligned}
\int_{\Omega} u^{\tau-\gamma} \psi^{2 m} & \leq C_{1} \int_{\Omega} u^{-\gamma+1}|\psi|^{2 m-2}\left[|\nabla \psi|^{2}+|\psi \Delta \psi|\right] \\
& \leq C_{1}\left(\int_{\Omega}\left(u^{-\gamma+1}|\psi|^{2 m-2}\right)^{\frac{\tau-\gamma}{1-\gamma}}\right)^{\frac{1-\gamma}{\tau-\gamma}}\left(\int_{\Omega}\left(|\nabla \psi|^{2}+|\psi||\Delta \psi|\right)^{\frac{\tau-\gamma}{\tau-1}}\right)^{\frac{\tau-1}{\tau-\gamma}} \\
& \leq C_{1}\left(\int_{\Omega} u^{\tau-\gamma}|\psi|^{2 m}\right)^{\frac{1-\gamma}{\tau-\gamma}}\left(\int_{\Omega}\left(|\nabla \psi|^{2}+|\psi||\Delta \psi|\right)^{\frac{\tau-\gamma}{\tau-1}}\right)^{\frac{\tau-1}{\tau-\gamma}} \\
& \leq C_{1}^{\frac{\tau-\gamma}{\tau-1}}\left(\int_{\Omega}\left(|\nabla \psi|^{2}+|\psi||\Delta \psi|\right)^{\frac{\tau-\gamma}{\tau-1}}\right) .
\end{aligned}
$$

This proves (2.4) with $C=C_{1}^{\frac{\tau-\gamma}{\tau-1}}$.

Now we prove (2.5). Combine (2.2) and (2.6) and we get for any $\varphi \in C_{c}^{2}(\Omega)$

$$
\begin{aligned}
& \int_{\Omega}\left|\nabla\left(u^{\frac{-\gamma+1}{2}}\right)\right|^{2} \varphi^{2} \\
\leq & \frac{(-\gamma+1)^{2}}{4 \gamma}\left[\frac{1}{\alpha} \int_{\Omega} u^{-\gamma+1}|\nabla \varphi|^{2}+\frac{\beta}{\alpha} \int_{\Omega} u^{-\gamma+1} \varphi \Delta \varphi\right]-\frac{-\gamma+1}{4 \gamma} \int_{\Omega} u^{-\gamma+1} \Delta \varphi^{2} \\
= & \frac{(-\gamma+1)^{2}}{4 \gamma}\left[\frac{1}{\alpha} \int_{\Omega} u^{-\gamma+1}|\nabla \varphi|^{2}+\frac{\beta}{\alpha} \int_{\Omega} u^{-\gamma+1} \varphi \Delta \varphi\right] \\
& \quad-\frac{-\gamma+1}{2 \gamma}\left[\int_{\Omega} u^{-\gamma+1}|\nabla \varphi|^{2}+\int_{\Omega} u^{-\gamma+1} \varphi \Delta \varphi\right] \\
= & A \int_{\Omega} u^{-\gamma+1}|\nabla \varphi|^{2}+B \int_{\Omega} u^{-\gamma+1} \varphi \Delta \varphi,
\end{aligned}
$$

where $A=\frac{(-\gamma+1)^{2}}{4 \gamma \alpha}-\frac{(-\gamma+1)}{2 \gamma}>0, B=\frac{(-\gamma+1)^{2} \beta}{4 \gamma \alpha}-\frac{(-\gamma+1)}{2 \gamma} \in \mathbb{R}$. We insert the test function $\varphi=\psi^{2 m}$ in the later inequality to get

$$
\begin{aligned}
\int_{\Omega}\left|\nabla\left(u^{\frac{-\gamma+1}{2}}\right)\right|^{2} \psi^{2 m} & \leq \int_{\Omega} u^{-\gamma+1} \psi^{2 m}\left[A m^{2}|\nabla \psi|^{2}+B m(m-1)|\nabla \psi|^{2}+B m \psi \Delta \psi\right] \\
& \leq C_{2} \int_{\Omega} u^{-\gamma+1}|\psi|^{2 m-2}\left[|\nabla \psi|^{2}+|\psi \Delta \psi|\right],
\end{aligned}
$$

with $C_{2}=\max \left\{\left|A m^{2}+B m(m-1)\right|,|B m|\right\}>0$. By using Hölder inequality in the above last inequality, we have

$$
\begin{aligned}
\int_{\Omega}\left|\nabla\left(u^{\frac{-\gamma+1}{2}}\right)\right|^{2} \psi^{2 m} & \leq C_{2}\left(\int_{\Omega}\left(u^{-\gamma+1}|\psi|^{2 m-2}\right)^{\frac{\tau-\gamma}{1-\gamma}}\right)^{\frac{1-\gamma}{\tau-\gamma}}\left(\int_{\Omega}\left(|\nabla \psi|^{2}+|\psi \Delta \psi|\right)^{\frac{\tau-\gamma}{\tau-1}}\right)^{\frac{\tau-1}{\tau-\gamma}} \\
& \leq C_{2}\left(\int_{\Omega} u^{\tau-\gamma}|\psi|^{2 m}\right)^{\frac{1-\gamma}{\tau-\gamma}}\left(\int_{\Omega}\left(|\nabla \psi|^{2}+|\psi \Delta \psi|\right)^{\frac{\tau-\gamma}{\tau-1}}\right)^{\frac{\tau-1}{\tau-\gamma}}
\end{aligned}
$$


In the above, we used again the facts that $|\psi|^{(2 m-2) \frac{\tau-\gamma}{1-\gamma}} \leq|\psi|^{2 m}$.

Finally, we insert (2.4) into the later inequality and obtain

$$
\int_{\Omega}\left|\nabla u^{\frac{-\gamma+1}{2}}\right|^{2} \psi^{2 m} \leq C_{2} C_{1}^{\frac{1-\gamma}{\tau-1}} \int_{\Omega}\left(|\nabla \psi|^{2}+|\psi||\Delta \psi|\right)^{\frac{\tau-\gamma}{\tau-1}} .
$$

This proves (2.5) with $C=C_{2} C_{1}^{\frac{1-\gamma}{\tau-1}}$.

Lemma 2.2. Suppose that $u$ is a positive solution of (2.1), which is stable outside a compact set of $\mathbb{R}^{\mathbb{N}}$, then there exists $R_{0}=R_{0}(u)>0$ such that

(i) for every $\gamma \in[1,1-2 \tau+2 \sqrt{\tau(\tau-1)})$ and every $r>R_{0}+3$, we have

$$
\int_{R_{0}+2<|x|<r}\left(\left|\nabla\left(u^{\frac{-\gamma+1}{2}}\right)\right|^{2}+u^{\tau-\gamma}\right) d x \leq A+B r^{N-2\left(\frac{\tau-\gamma}{\tau-1}\right)},
$$

where $A, B$ are positive constants depending on $\tau, \gamma, N, R_{0}$ but not $r$.

(ii) for every $\gamma \in[1,1-2 \tau+2 \sqrt{\tau(\tau-1)})$ and every open ball $B_{R}(y)$ centered at $y$ with radius $R$ such that $B_{2 R}(y) \subset\left\{x \in \mathbb{R}^{\mathbb{N}}:|x|>R_{0}\right\}$, we have

$$
\int_{R_{0}+2<|x|<r}\left(\left|\nabla\left(u^{\frac{-\gamma+1}{2}}\right)\right|^{2}+u^{\tau-\gamma}\right) d x \leq C R^{N-2\left(\frac{\tau-\gamma}{\tau-1}\right)},
$$

where $C$ is a positive constant independent of $R$ and $y$.

Proof. Since $u$ is stable outside a compact set of $\mathbb{R}^{\mathbb{N}}$. There exists a $R_{0}>0$ such that Proposition 2.1 is true with $\Omega=\mathbb{R}^{\mathbb{N}} \backslash \overline{B_{R_{0}}(0)}$. We choose $\varphi \in C_{c}^{2}(\mathbb{R})$ satisfying $0 \leq \varphi \leq 1$ everywhere on $\mathbb{R}$ and

$$
\varphi(t)=\left\{\begin{array}{lll}
1 & \text { if } & |t| \leq 1 \\
0 & \text { if } & |t| \geq 2
\end{array}\right.
$$

for $s>0$, we take a function $\theta_{s} \in C_{c}^{2}(\mathbb{R})$ such that $0 \leq \theta_{s} \leq 1$ and

$$
\theta_{s}(t)=\left\{\begin{array}{lll}
0 & \text { if } & |t| \leq s+1 \\
1 & \text { if } & |t| \geq s+2 .
\end{array}\right.
$$

Now for every $r>R_{0}+3$, we define a function $\xi_{r}(x) \in C_{c}^{2}\left(\mathbb{R}^{\mathbb{N}}\right)$ by

$$
\xi_{r}(x)=\left\{\begin{array}{lll}
\theta_{R_{0}}(|x|) & \text { if } & |x| \leq R_{0}+3 \\
\varphi\left(\frac{|x|}{r}\right) & \text { if } & |x| \geq R_{0}+3
\end{array}\right.
$$

then one can easily see that $\xi_{r} \in C_{c}^{2}\left(\mathbb{R}^{\mathbb{N}} \backslash \overline{B_{R_{0}}(0)}\right)$ and satisfies $0 \leq \xi_{r} \leq 1$ on $\mathbb{R}^{\mathbb{N}}$. We choose $m=1+\operatorname{INT}\left[\max \left\{\frac{\tau-\gamma}{\tau-1}, 2\right\}\right]$, where INT $[s]$ denotes the integer part of the real number $s$. We apply Proposition 2.1 with $\Omega=\mathbb{R}^{\mathbb{N}} \backslash \overline{B_{R_{0}}(0)}$ and obtain:

$$
\begin{aligned}
& \int_{\left\{R_{0}+2<|x|<r\right\}}\left(\left|\nabla\left(u^{\frac{-\gamma+1}{2}}\right)\right|^{2}+u^{\tau-\gamma}\right) d x \\
\leq & C(\tau, m, \gamma) \int_{\left\{R_{0}+2<|x|<r\right\}}\left(\left|\nabla \xi_{r}\right|^{2}+\left|\xi_{r}\right|\left|\Delta \xi_{r}\right|\right)^{\frac{\tau-\gamma}{\tau-1}} d x \\
\leq & C(\tau, m, \gamma)\left[\int_{|x| \leq R_{0}+3}\left(\left|\nabla \theta_{R_{0}}\right|^{2}+\left|\theta_{R_{0}}\right|\left|\Delta \theta_{R_{0}}\right|\right)^{\frac{\tau-\gamma}{\tau-1}}\right. \\
& \left.\quad+\int_{\{r \leq|x| \leq 2 r\}}\left(\left|\nabla \xi_{r}\right|^{2}+\left|\xi_{r}\right|\left|\Delta \xi_{r}\right|\right)^{\frac{\tau-\gamma}{\tau-1}}\right] \\
\leq & C_{1}\left(\tau, \gamma, m, N, \theta, R_{0}\right)+C_{2}(\tau, \gamma, m, N, \varphi) r^{N-2\left(\frac{\tau-\gamma}{\tau-1}\right)} d x,
\end{aligned}
$$


for all $r>R_{0}+3$ and all $\gamma \in[1,1-2 \tau+2 \sqrt{\tau(\tau-1)})$, hence the desired estimate (i) follows.

To prove (ii), we fix $m=1+\operatorname{INT}\left[\max \left\{\frac{\tau-\gamma}{\tau-1}, 2\right\}\right]$, and we take the test function $\psi$ in Proposition 2.1 as $\psi_{R, y}=\varphi\left(\frac{|x-y|}{R}\right)$, it leads to

$$
\begin{aligned}
\int_{B_{R}(y)}\left(\left|\nabla\left(u^{\frac{-\gamma+1}{2}}\right)\right|^{2}+u^{\tau-\gamma}\right) & \leq C(\tau, m, \gamma) \int_{\mathbb{R}^{\mathbb{N}}}\left(\left|\nabla \psi_{R, y}\right|^{2}+\left|\psi_{R, y}\right|\left|\Delta \psi_{R, y}\right|\right)^{\frac{\tau-\gamma}{\tau-1}} \\
& =C(\tau, m, \gamma) \int_{B_{2 R}(0)}\left(\left|\nabla \psi_{R_{0}, 0}\right|^{2}+\left|\psi_{R_{0}, 0}\right|\left|\Delta \psi_{R_{0}, 0}\right|\right)^{\frac{\tau-\gamma}{\tau-1}} \\
& \leq C(\tau, m, \gamma, N, \varphi) R^{N-2\left(\frac{\tau-\gamma}{\tau-1}\right)} .
\end{aligned}
$$

And this completes the proof of the estimates (ii).

3. Proof of the Theorem 1.2 with $\alpha=0$. With the help of Proposition 2.1 and Lemma 2.2, in this section we are concerned with the proof of Theorem 1.2 with $\alpha=0$. We first prove the following:

Lemma 3.1. Let $\eta>0$ and $R_{0}$ be chosen as in Lemma 2.2. Assume $u$ is a solution which is stable outside a compact set in $\mathbb{R}^{\mathbb{N}}$, if $2<N<10, \tau<P_{c}:=$ $\frac{(N-2)^{2}-4 N+8 \sqrt{N-1}}{(N-10)(N-2)}$, then $\exists R_{1}=R_{1}(\tau, N, \eta, u)>R_{0}$ such that

(i) $\int_{|x| \geq R_{1}} u^{(\tau-1) \frac{N}{2}}<\eta$,

(ii) There are constants $C_{1}$ and $C_{2}$ such that $C_{1}|x|^{-\frac{2}{\tau-1}} \leq u(x) \leq C_{2}\left(1+|x|^{-\frac{2 \tau}{\tau-1}}\right)$.

Proof. We first prove (i). We observe that $2 \leq N$ implies

$$
(\tau-1) \frac{N}{2} \leq(\tau-1) .
$$

On the other hand, let $\gamma_{M}=1-2 \tau+\sqrt{\tau(\tau-1)}$, then $\lim _{\tau \rightarrow-\infty} \frac{\tau-\gamma_{M}}{\tau-1}=5$ and $\lim _{\tau \rightarrow 0^{-}} \frac{\tau-\gamma_{M}}{\tau-1}=1$, thus there must be a number $\tau_{0}$ such that $\frac{N}{2}=\frac{\tau_{0}-\gamma_{M}\left(\tau_{0}\right)}{\tau_{0}-1}$. We claim $\tau_{0}=P_{c}$. Indeed, a direct computation shows that $\tau_{0}$ satisfies

$$
(N-10)(N-2) \tau_{0}^{2}-2\left[N^{2}-8 N+4\right] \tau_{0}+(N-2)^{2}=0 .
$$

The roots of the above equations are

$$
\tau_{1,2}=\frac{(N-2)^{2}-4 N \pm 8 \sqrt{N-1}}{(N-10)(N-2)} .
$$

Since $\tau_{0}<0$ and $\frac{(N-2)^{2}-4 N-8 \sqrt{N-1}}{(N-10)(N-2)}>0$, we get $\tau_{0}=P_{c}$. Note that the function $\frac{\tau-\gamma}{\tau-1}$ is strictly decreasing in $\tau$, it follows that

$$
\left(\tau-\gamma_{M}\right) \leq(\tau-1) \frac{N}{2}, \text { for } \tau \leq P_{c} .
$$

At last, combine the inequalities (3.1) and (3.2) together with the continuity of the function $f(\gamma)=(\tau-1) \frac{N}{2}-(\tau-\gamma)$, we obtain that there exists $\gamma_{1} \in[1,1-2 \tau+$ $2 \sqrt{\tau(\tau-1)})$ such that $(\tau-1) \frac{N}{2}=\tau-\gamma_{1}$. Moreover the continuity of the function $g(\gamma, \epsilon)=(\tau-1) \frac{N}{2-\epsilon}-(\tau-\gamma)$ implies that $\exists \epsilon \in(0,1]$ such that

$$
1 \leq(\tau-1) \frac{N}{2-\epsilon}-\tau<1-2 \tau+2 \sqrt{\tau(\tau-1)} .
$$


Hence by Lemma 2.2 (i), we have

$$
\begin{aligned}
\int_{\left\{R_{0}+2<|x|<r\right\}} u^{(\tau-1) \frac{N}{2}} d x & =\int_{\left\{R_{0}+2<|x|<r\right\}} u^{\tau-\gamma_{1}} d x \\
& \leq A+B r^{N-2\left(\frac{\tau-\gamma_{1}}{\tau-1}\right)} \\
& =A+B r^{0}, \text { for } r>R_{0}+3,
\end{aligned}
$$

thus

$$
\int_{\left\{|x| \geq R_{0}+2\right\}} u^{(\tau-1) \frac{N}{2}}<+\infty
$$

Therefore there exists $R_{1}=R_{1}(\tau, N, \eta, u)>R_{0}$ such that for any $\eta>0$,

$$
\int_{\left\{|x| \geq R_{1}\right\}} u^{(\tau-1) \frac{N}{2}}<\eta \text {. }
$$

In order to prove (ii), we will need the following known results due to Gibarg and Trudinger, see also Han [17].

LEMma 3.2 Let $u \in H_{0}^{1}(\Omega)$ be a positive smooth subsolution of

$$
-\Delta u=d(x) u,
$$

with $d(x) \in L^{\alpha}(\Omega)$ for $\alpha>\frac{N}{2}$. Then for any $y \in \mathbb{R}^{\mathbb{N}}$,

$$
\sup _{B_{R}(y)} \leq C\left[R^{-N} \int_{B_{2 R}(y)} u^{p}\right]^{\frac{1}{p}}
$$

where $C$ is dependent on $|d(x)|_{L^{\infty}(\Omega)}$ and $\alpha, N$.

Proof. This is more or less standard elliptic regularity, see Theorem 8.17 in [16].

Now we consider points $y \in \mathbb{R}^{\mathbb{N}}$ such that $|y|>10 R_{1}$, and let $R=\frac{|y|}{4}$. Where $R_{1}>R_{0}$, and obviously we have

$$
B_{2 R}(y) \subset\left\{x \in \mathbb{R}^{\mathbb{N}}:|x|>R_{1}\right\} \subset\left\{x \in \mathbb{R}^{\mathbb{N}}:|x|>R_{0}\right\} .
$$

Observe that from (3.3) there exist $\epsilon \in(0,1]$ such that $\exists \gamma_{2} \in[1,1-2 \tau+\sqrt{\tau(\tau-1)})$ such that $(\tau-1) \frac{N}{2-\epsilon}=\tau-\gamma_{2}$. We fix $\eta>0$, and let $w=\frac{1}{u}$, then we have

$$
\Delta w=\left[2 \frac{|\nabla u|^{2}}{u^{2}}-u^{\tau-1}\right] w .
$$

It deduces that

$$
\Delta w+u^{\tau-1} w \geq 0 .
$$

We apply Lemma 3.2 to the above inequality equation with $d(x)=u^{\tau-1}$ and $p=2$. Note that $|d(x)| \in L^{\frac{N}{2-\epsilon}}\left(B_{2 R}(y)\right)$. Thus

$$
\sup _{B_{R}(y)}|w| \leq C\left(R^{-\frac{N}{2}}\right)\|w\|_{L^{2}\left(B_{2 R}(y)\right)} .
$$


Using Hölder inequality, we get

$$
\begin{aligned}
\sup _{B_{R}(y)} w & \leq C_{1} R^{\frac{N}{(\tau-1) \frac{N}{2}}}\|w\|_{L^{-(\tau-1) \frac{N}{2}}}\left(B_{2 R}(y)\right) \\
& =C_{1} R^{\frac{2}{\tau-1}}\|w\|_{L^{-(\tau-1) \frac{N}{2}}}\left(B_{2 R}(y) .\right.
\end{aligned}
$$

It follows from (i) that $\|w\|_{L^{-(\tau-1)} \frac{N}{2}}\left(B_{2 R}(y) \leq \eta\right.$, insert this result into the above last inequality, we obtain $|w(y)| \leq C_{1} R^{\frac{2}{\tau-1}} \eta=C_{2}|y|^{\frac{2}{\tau-1}}$, this implies that $u(x) \geq$ $C|x|^{-\frac{2}{\tau-1}}$. On the other hand by Theorem 18 in [22], we have $u(x) \geq C\left(1+|x|^{-\frac{2 \tau}{\tau-1}}\right)$. This completes the proof of Lemma 3.1.

Proof of Theorem 1.2. We prove the theorem by contrary. We perform the following Emden-fowler transformation

$$
v(t, \theta)=r^{\frac{2}{\tau-1}} u(r, \theta), t=\ln r, r=|x|,
$$

then $v$ satisfies the following equation

$$
v_{t t}+A v_{t}+\Delta_{S^{N-1}} v+B v-v^{\tau}=0 \quad \text { in } \mathbb{R} \times S^{N-1},
$$

where $A=\left(N-2-\frac{4}{\tau-1}\right), B=-\left[\frac{2}{\tau-1}\left(N-2-\frac{2}{\tau-1}\right)\right]$ and $S^{N-1}$ is the unit sphere of $\mathbb{R}^{\mathbb{N}}, \Delta_{S^{N-1}}$ denotes the Laplace-Beltrami operator on $S^{N-1}$. By Lemma 3.1 (ii), we conclude that $v(t, \theta) \geq C$.

We state the following claim whose proof will be given later.

Claim. $v(t, \theta) \leq C$.

By the results of L. Simon [23], we have $v(t, \theta) \rightarrow V(\theta)$, and $v(\theta)$ satisfies

$$
\Delta_{S^{N-1}} v+B v-v^{\tau}=0 .
$$

We consider the following ODE, namely

$$
\psi_{t t}+A \psi_{t}+(1-\tau) B \psi=0
$$

Then a simple calculation implies that $A^{2}-4(1-\tau) B<0$ provided that $2 \leq N<$ $2+\frac{4}{\tau-1}(\tau-\sqrt{\tau(\tau-1)})$. Thus one can easily deduce that the solution $\psi$ of the equation (3.4) has infinitely many zero points $T_{i}, i=1,2, \ldots$. We choose two neighborhoods of such $T_{i}, i=1,2, \ldots$, namely $T_{i}, T_{i+1}$ such that $R_{i}=e^{T_{i}}, R_{i+1}=e^{T_{i+1}}$ large enough (at least larger than $R_{0}$ ). Suppose $\varphi_{i}$ is the solution of the following problem:

$$
\Delta \varphi_{i}+\frac{\tau B}{r^{2}} \varphi_{i}=0, \varphi_{i}\left(R_{i}\right)=\varphi_{i}\left(R_{i+1}\right)=0 .
$$

Let $\psi_{i}(x)$ be the function defined to be $\varphi_{i}$ for $|x|$ between $R_{i}$ and $R_{i+1}$ and to be zero otherwise, then $\psi_{i} \in C_{c}^{2}\left(\mathbb{R}^{\mathbb{N}} \backslash B_{R}(0)\right)$ and $\psi_{i}$ are orthogonal in $L^{2}\left(\mathbb{R}^{\mathbb{N}}\right)$, using the fact that $u=r^{-\frac{2}{\tau-1}} v+o(1)$, we have

$$
\begin{aligned}
E\left(\psi_{i}\right) & =\int_{\mathbb{R}^{\mathbb{N}} \backslash B_{R}(0)}\left|\nabla \psi_{i}\right|^{2}+\tau \int_{\mathbb{R}^{\mathbb{N}} \backslash B_{R}(0)} u^{\tau-1} \psi_{i}^{2} \\
& =\int_{\mathbb{R}^{\mathbb{N}} \backslash B_{R}(0)}\left|\nabla \psi_{i}\right|^{2}+\tau \int_{\mathbb{R}^{\mathbb{N}} \backslash B_{R}(0)} r^{-2} v^{\tau-1} \psi_{i}^{2}+o(1) \\
& =\int_{\mathbb{R}^{\mathbb{N}} \backslash B_{R}(0)}\left|\nabla \psi_{i}\right|^{2}+\int_{R_{i}}^{R_{i+1}} \frac{\psi_{i}^{2}}{r^{2}} \int_{S^{N-1}} \tau v^{\tau-1}+o(1) .
\end{aligned}
$$


Now we multiply the equation of $v: \Delta_{S^{N-1}} v+B v-v^{\tau}=0$ by $v^{-1}$ and integrate by parts to find

$$
\int_{S^{N-1}} v^{\tau-1}=\int_{S^{N-1}} \frac{|\nabla v|^{2}}{v^{2}}+B\left|S^{N-1}\right| \geq B\left|S^{N-1}\right|,
$$

insert this result into the above last equality we obtain

$$
\begin{aligned}
E\left(\psi_{i}\right) & =\int_{\mathbb{R}^{\mathbb{N}} \backslash B_{R}(0)}\left|\nabla \psi_{i}\right|^{2}+\tau \int_{\mathbb{R}^{\mathbb{N}} \backslash B_{R}(0)} u^{\tau-1} \psi_{i}^{2} \\
& =\int_{\mathbb{R}^{\mathbb{N}} \backslash B_{R}(0)}\left|\nabla \psi_{i}\right|^{2}+\int_{R_{i}}^{R_{i+1}} \frac{\psi_{i}^{2}}{r^{2}} \int_{S^{N-1}} \tau v^{\tau-1}+o(1) \\
& <\int_{\mathbb{R}^{\mathbb{N}} \backslash B_{R}(0)}\left|\nabla \psi_{i}\right|^{2}+\int_{R_{i}}^{R_{i+1}} \frac{\psi_{i}^{2}}{r^{2}} \int_{S^{N-1}} \tau B+o(1) \\
& =\int_{R_{i}}^{R_{i+1}}\left|\nabla \psi_{i}\right|^{2}\left|S^{N-1}\right|+\int_{R_{i}}^{R_{i+1}} \tau B \frac{\psi_{i}^{2}}{r^{2}}\left|S^{N-1}\right|+o(1) \\
& =\left|S^{N_{1}}\right| \int_{R_{i}}^{R_{i+1}}\left(\left|\nabla \psi_{i}\right|^{2}+\frac{\tau B}{r^{2}} \psi_{i}^{2}\right)+o(1) \\
& =0 .
\end{aligned}
$$

This shows that

$$
E\left(\psi_{i}\right)<0 .
$$

Since there are infinitely many of such $T_{i}, i=1,2, \ldots$, consequently we can choose infinitely many of such test function $\psi_{i} \in C_{c}^{2}\left(\mathbb{R}^{\mathbb{N}} \backslash B_{R}(0)\right)$ and $\psi_{i}$ are orthogonal in $L^{2}\left(\mathbb{R}^{\mathbb{N}}\right)$ such that

$$
E\left(\psi_{i}\right)=\int\left|\nabla \psi_{i}\right|^{2}+\tau \int u^{\tau-1} \psi_{i}^{2}<0
$$

and hence the Morse index of $u$ must be infinity, a contradiction with our assumption.

Finally, we prove the Claim. In fact, suppose the claim is not true. Then there exists $\left(t_{i}, \theta_{i}\right)$ such that $v\left(t_{i}, \theta_{i}\right) \rightarrow+\infty$. Since $v$ satisfies a linear equation

$$
v_{t t}+A v_{t}+\Delta_{S^{N-1}} v+V v=0 \quad \text { in } \mathbb{R} \times S^{N-1},
$$

where $|V| \leq C$. By Harnack inequality, we can find $\delta$ small, and $T$ large such that $v^{\tau-1}(t, \theta)<\delta$ for $t \in\left[t_{i}, t_{i}+T\right]$.

We complete the proof of Theorem 1.2 with $\alpha=0$.

4. Proof of Theorem 1.2 with $\alpha>0$. In this section, we are concerned with the more general equation:

$$
\Delta u=|x|^{\alpha} u^{\tau}, \alpha>0, \tau<0 \text { in } \mathbb{R}^{\mathbb{N}} .
$$

Since we are considering the solution which is stable outside a compact set in $\mathbb{R}^{\mathbb{N}}$. The critical step is to prove the following integral inequality:

Lemma 4.1. Let $\eta>0$ and $R_{0}$ be chosen large enough. Assume that $u$ is a solution of $(4.1)$, which is stable outside a compact set in $\mathbb{R}^{\mathbb{N}}$. If $2 \leq N<2+$ $\frac{4+2 \alpha}{\tau-1}(\tau-\sqrt{\tau(\tau-1)})$, then $\exists R_{1}=R_{1}(\tau, N, \eta, u, \alpha)>R_{0}$ such that 
(i) $\int_{|x| \geq R_{1}}|x|^{\frac{N}{2} \alpha} u^{(\tau-1) \frac{N}{2}}<\eta$.

(ii) There exists constant $C$ such that $u(x) \geq C|x|^{-\frac{2+\alpha}{\tau-1}}$.

Proof. Let $\hat{u}=R^{\frac{\alpha}{\tau-1}} u$, then $\hat{u}$ satisfies the following equation

$$
\Delta \hat{u}=\left(\frac{|x|}{R}\right)^{\alpha} \hat{u}^{2}
$$

Since we are going to consider a solution which is sable outside of a compact set in $\mathbb{R}^{\mathbb{N}}$, it is sufficient to consider the equation in an annul domain, that $C_{1} R \leq|x| \leq C_{2} R$. Hence, up to a constant, we apply Lemma 3.1 to the equation (4.2) to get:

(i) $\int_{|x| \geq R_{1}} \hat{u}^{(\tau-1) \frac{N}{2}}<\eta$.

(ii) There exists constant $C$ such that $\hat{u}(x) \geq C|x|^{-\frac{2}{\tau-1}}$.

Note that $\hat{u}=R^{\frac{\alpha}{\tau-1}} u$, and $R=C|x|$, the desired inequalities follows immediately.

Proof of Theorem 1.2 with $\alpha>0$. The idea is the same as in the case of $\alpha=0$ but with necessary modifications. We sketch it below. We perform the following Emden-fowler transformation

$$
v(t, \theta)=r^{\frac{2+\alpha}{\tau-1}} u(r, \theta), t=\ln r, r=|x|,
$$

then $v$ satisfies the following equation

$$
v_{t t}+\tilde{A} v_{t}+\Delta_{S^{N-1}} v+\tilde{B} v-v^{\tau}=0 \quad \text { in } \mathbb{R} \times S^{N-1},
$$

where $\tilde{A}=\left(N-2-\frac{4+2 \alpha}{\tau-1}\right), \tilde{B}=-\left[\frac{2+\alpha}{\tau-1}\left(N-2-\frac{2+\alpha}{\tau-1}\right)\right]$ and $S^{N-1}$ is the unit sphere of $\mathbb{R}^{\mathbb{N}}, \Delta_{S^{N-1}}$ denotes the Laplace-Beltrami operator on $S^{N-1}$. By Lemma 4.1 (ii), we conclude that $v(t, \theta) \geq C$. And by the same arguments as in Section 3, we get $v(t, \theta) \leq C$. Hence By the results of L.Simon [23], we have $v(t, \theta) \rightarrow V(\theta)$, and $v(\theta)$ satisfies

$$
\Delta_{S^{N-1}} v+\tilde{B} v-v^{\tau}=0 .
$$

We consider the following ODE, namely

$$
\psi_{t t}+\tilde{A} \psi_{t}+(1-\tau) \tilde{B} \psi=0
$$

Then a simple calculation implies that $\tilde{A}^{2}-4(1-\tau) \tilde{B}<0$ provided that $2 \leq N<2+$ $\frac{4+2 \alpha}{\tau-1}(\tau-\sqrt{\tau(\tau-1)})$. Thus one can easily deduce that the solution $\psi$ of the equation (4.3) has infinitely many zero points $T_{i}, i=1,2, \ldots$. We choose two neighborhoods of such $T_{i}, i=1,2, \ldots$, namely $T_{i}, T_{i+1}$ such that $R_{i}=e^{T_{i}}, R_{i+1}=e^{T_{i+1}}$ large enough (at least larger than $R_{0}$ ). Suppose $\varphi_{i}$ is the solution of the following problem:

$$
\Delta \varphi_{i}+\frac{\tau \tilde{B}}{r^{2}} \varphi_{i}=0, \varphi_{i}\left(R_{i}\right)=\varphi_{i}\left(R_{i+1}\right)=0 .
$$

Let $\psi_{i}(x)$ be the function defined to be $\varphi_{i}$ for $|x|$ between $R_{i}$ and $R_{i+1}$ and to be zero otherwise, then $\psi_{i} \in C_{c}^{2}\left(\mathbb{R}^{\mathbb{N}} \backslash B_{R}(0)\right)$ and $\psi_{i}$ are orthogonal in $L^{2}\left(\mathbb{R}^{\mathbb{N}}\right)$, using the fact that $u=r^{-\frac{2+\alpha}{\tau-1}} v+o(1)$, we have 


$$
\begin{aligned}
E\left(\psi_{i}\right) & =\int_{\mathbb{R}^{\mathbb{N}} \backslash B_{R}(0)}\left|\nabla \psi_{i}\right|^{2}+\tau \int_{\mathbb{R}^{\mathbb{N}} \backslash B_{R}(0)}|x|^{\alpha} u^{\tau-1} \psi_{i}^{2} \\
& =\int_{\mathbb{R}^{\mathbb{N}} \backslash B_{R}(0)}\left|\nabla \psi_{i}\right|^{2}+\tau \int_{\mathbb{R}^{\mathbb{N}} \backslash B_{R}(0)} r^{-2} v^{\tau-1} \psi_{i}^{2}+o(1) \\
& =\int_{\mathbb{R}^{\mathbb{N}} \backslash B_{R}(0)}\left|\nabla \psi_{i}\right|^{2}+\int_{R_{i}}^{R_{i+1}} \frac{\psi_{i}^{2}}{r^{2}} \int_{S^{N-1}} \tau v^{\tau-1}+o(1) .
\end{aligned}
$$

Now we multiply the equation of $v: \Delta_{S^{N-1}} v+\tilde{B} v-v^{\tau}=0$ by $v^{-1}$ and integrate by parts to find

$$
\int_{S^{N-1}} v^{\tau-1}=\int_{S^{N-1}} \frac{|\nabla v|^{2}}{v^{2}}+\tilde{B}\left|S^{N-1}\right|>\tilde{B}\left|S^{N-1}\right|,
$$

insert this result into the above last equality we obtain

$$
\begin{aligned}
E\left(\psi_{i}\right) & =\int_{\mathbb{R}^{\mathbb{N}} \backslash B_{R}(0)}\left|\nabla \psi_{i}\right|^{2}+\tau \int_{\mathbb{R}^{\mathbb{N}} \backslash B_{R}(0)}|x|^{\alpha} u^{\tau-1} \psi_{i}^{2} \\
& =\int_{\mathbb{R}^{\mathbb{N}} \backslash B_{R}(0)}\left|\nabla \psi_{i}\right|^{2}+\int_{R_{i}}^{R_{i+1}} \frac{\psi_{i}^{2}}{r^{2}} \int_{S^{N-1}} \tau v^{\tau-1}+o(1) \\
& <\int_{\mathbb{R}^{\mathbb{N}} \backslash B_{R}(0)}\left|\nabla \psi_{i}\right|^{2}+\int_{R_{i}}^{R_{i+1}} \frac{\psi_{i}^{2}}{r^{2}} \int_{S^{N-1}} \tau \tilde{B}+o(1) \\
& =\int_{R_{i}}^{R_{i+1}}\left|\nabla \psi_{i}\right|^{2}\left|S^{N-1}\right|+\int_{R_{i}}^{R_{i+1}} \tau \tilde{B} \frac{\psi_{i}^{2}}{r^{2}}\left|S^{N-1}\right|+o(1) \\
& =\left|S^{N_{1}}\right| \int_{R_{i}}^{R_{i+1}}\left(\left|\nabla \psi_{i}\right|^{2}+\frac{\tau \tilde{B}^{2}}{r^{2}} \psi_{i}^{2}\right)+o(1) \\
& =0 .
\end{aligned}
$$

This shows that

$$
E\left(\psi_{i}\right)<0 .
$$

Since there are infinitely many of such $T_{i}, i=1,2, \ldots$, consequently we can choose infinitely many of such test function $\psi_{i} \in C_{c}^{2}\left(\mathbb{R}^{\mathbb{N}} \backslash B_{R}(0)\right)$ and $\psi_{i}$ are orthogonal in $L^{2}\left(\mathbb{R}^{\mathbb{N}}\right)$ such that

$$
E\left(\psi_{i}\right)=\int\left|\nabla \psi_{i}\right|^{2}+\tau \int|x|^{\alpha} u^{\tau-1} \psi_{i}^{2}<0,
$$

and hence the Morse index of $u$ must be infinity, a contradiction with our assumption. We complete the proof.

\section{REFERENCES}

[1] A. BAhri And P. L. Lions, Solutions of superlinear elliptic equations and their Morse indices, Comm. Pure. Appl. Math., 45:9 (1992), pp. 1205-1215.

[2] A. L. Bertozzi And M. C. Pugh, Long-wave instabilities and staturation in thin film equations, Comm. Pure. Appl. Math., 51:6 (1998), pp. 625-661.

[3] A. L. Bertozzi and M. C. Pugh, Finite-time blow-up of solutions of some longwave unstable thin film equations, Indiana Univ. Math. J., 49 (2000), pp. 1323-1366.

[4] E. N. DANCER AND A. FARINA, On the classification of the solutions $-\Delta u=e^{u}$ in $R^{N}$ : stability outside a compact set and applications, Proc. Amer. Math. Soc., 137 (2009), pp. 1333-1338. 
[5] P. Esposito, Compactness of a nonlinear eigenvalue problem with a singular nonlinearity, Comm. Contemp. Math., 10 (2008), pp. 17-45.

[6] P. Esposito, N. Ghoussoub And Y. Guo, Compactness along the branch of semi-stable and unstable solutions for an elliptic problem with a singular nonlinearity, Comm. Pure Appl. Math., 60 (2007), pp. 1731-1768.

[7] A. FARINA, On the classification ofsolutions of Lane-Emden equation on unbounded domains of $\mathbb{R}^{\mathbb{N}}$, J. Math. Pures Appl., 87:9 (2007), pp. 537-561.

[8] D. G. DE Figueiredo, Semilimear elliptic systems, Research surve, Universidade Estadual de Campinas, 1998.

[9] N. Ghoussoub And Y. Guo, On the partial differential equations of electrostatic MEMS devices II: dynamic case, NoDEA Nonlinear Diff. Equations Appl., 15 (2008), pp. 115-145.

[10] N. Ghoussoub and Y. Guo, On the partial differential equations of electrostatic MEMS devices: stationary case, preprint.

[11] Y. Guo, On the partial differential equations of electrostatic MEMS devices III: refined touchdown behavior, J. Differential Equations, 244:9 (2008), pp. 2277-2309.

[12] B. Gidas And J. SpRUCK, A priori bounds for positive solutions of semilinear elliptic equations, Comm. Partial Differential Equations, 6:8 (1981), pp. 883-901.

[13] Z. M. GUO AND J. C. WEI, Infinitely many turning points for an elliptic problem with a singular nonlinearity, J. Lond. Math. Soc., 78:2 (2008), pp. 21-35.

[14] Z. M. Guo AND J. C. WeI, Symmetry of nonnegative solutions of asemilinear elliptic equation with singular nonlinearity, Proc.R.Soc. Edinb., 137 A (2007), pp. 963-994.

[15] Z. M. Guo AND J. C. WeI, Hausdorff dimension of ruptures for solutions of a semilinear elliptic equation with singular nonlinearity, Manuscripta Math., 120 (2006), pp. 193-209.

[16] Gilbarg and N. Trudinger, Elliptic Equations of Second Order, Springer-Verlag.

[17] Z. C. HAN, Asymptotic approach to singular solutions for nonlinear elliptic equations involving critical soblev exponent, Ann. Inst. Henri Poincare, 8 (1991), pp. 159-174.

[18] H. Q. JIANG AND W. M. NI, On steady states of Van Der Waals force drive thin film equations, arXiv: Math/ 0610762.

[19] R. S. Laugesen and M. C. Pugh, Properties of steady states for thin film equations, Eur. J. Appl. Math., 11 (2000), pp. 293-351.

[20] R. S. Laugesen And M. C. Pugh, Linear stability of steady states for thin film and CahnHilliard type equations, Arch. Ration. Mech. Anal., 154 (2000), pp. 13-51

[21] A. Meadows, Stable and singular solutions of the equation $\Delta u=\frac{1}{u}$, Indiana Univ. Math. J., 53 (2004), pp. 1419-1430.

[22] L. Ma And J. C. WeI, Properties of positive solutions to an elliptic equation with negative exponent, J. Funct. Anal., 2007.

[23] L. Simon, Asymptotics for a class of nonlinear evolution equations with applications to geometric problems, Ann. Math., 118 (1983), pp. 525-571.

[24] L. Simon, Some examples of singular minimal hypersurface, 2001. 
